\title{
Lower Sympathetic Nervous System Density and $\beta$-adrenoreceptor Expression Are Involved in Gastric Cancer Progression
}

\author{
GO EUN BAE ${ }^{1,2 *}$, HYUN-SOO KIM $^{3 *}$, KYU YEOUN WON $^{1}$, \\ GOU YOUNG KIM ${ }^{1}$, JI-YOUN SUNG ${ }^{4}$ and SUNG-JIG LIM ${ }^{1}$ \\ ${ }^{1}$ Department of Pathology, Kyung Hee University Hospital at Gangdong, \\ Kyung Hee University School of Medicine, Seoul, Republic of Korea; \\ ${ }^{2}$ Department of Pathology, Chungnam National University School of Medicine, Daejeon, Republic of Korea; \\ ${ }^{3}$ Department of Pathology, Severance Hospital, \\ Yonsei University College of Medicine, Seoul, Republic of Korea; \\ ${ }^{4}$ Department of Pathology, Kyung Hee University Hospital, \\ Kyung Hee University School of Medicine, Seoul, Republic of Korea
}

\begin{abstract}
Background/Aim: Identifying the role of the sympathetic nervous system (SNS) in tumor progression is among the most important challenges in cancer research. This study aimed to investigate the role of the SNS and $\beta$-adrenoreceptor in gastric cancer progression. Materials and Methods: The density of SNS was quantified by immunohistochemical staining for tyrosine hydroxylase in 115 surgically-resected gastric cancer specimens. Immunostaining for $\beta 1$ - and $\beta 2$-adrenoreceptor was also performed to examine the $\beta$-adrenoreceptor expression status in gastric cancer. Then the association of protein expression status with histological grade, pathological tumor stage $(p T)$, and pathological node stage of gastric cancer was investigated. Results: The SNS density of pT4 tumors was significantly lower than that of pT1-3 tumors. The SNS density was positively correlated with $\beta 1$-adrenoreceptor expression status. In addition, lower $\beta 1$-adrenoreceptor expression was significantly associated with increased lymph node metastasis. Reduced $\beta 2$-adrenoreceptor staining proportion was significantly associated with worse histological grade. Furthermore, the proportion of $\beta 2$ -
\end{abstract}

\footnotetext{
*These Authors contributed equally to this study.

Correspondence to: Dr. Sung-Jig Lim, Department of Pathology, Kyung Hee University Hospital at Gangdong, Kyung Hee University School of Medicine, 892, Dongnam-ro, Gangdong-gu, 05278 Seoul, Republic of Korea. Tel: +82 24405505/+82 24406434, e-mail: sungjig@khu.ac.kr
}

Key Words: Stomach, gastric cancer, sympathetic nervous system, $\beta$-adrenoreceptor. adrenoreceptor staining was significantly lower in tumors with diffuse-type histology, than those with intestinal-type histology. Conclusion: A lower SNS density and $\beta$ adrenoreceptor expression was associated with an aggressive oncogenic behavior including worse histological grade, advanced pT, and increased lymph node metastasis. SNS and $\beta$-adrenergic pathway are involved in the negative regulation of gastric cancer progression.

The sympathetic nervous system (SNS) regulates the function of all human organ systems through localized release of catecholamine from sympathetic nerve terminals and through systemic circulation of catecholamine from the adrenal gland (1). SNS signaling exerts clinically significant effects on tumor biology (2-4). In particular, SNS activation can regulate gene expression and cellular function in the tumor microenvironment (1). The $\beta$-adrenergic pathway mediates the SNS (4), and the $\beta$-adrenergic receptor ( $\beta$-AR) system has been reported to be involved in tumor progression $(5,6)$. Preclinical studies have shown that $\beta$-adrenergic antagonists inhibited distant metastasis, whereas $\beta$-adrenergic agonists accelerated tumor progression even in the absence of stress (7-10). These data suggest that SNS signaling contributes to progression and metastasis of malignant tumors.

Gastric cancer is the fifth most common cancer worldwide and is among the cancers with poor prognosis, with a 5-year survival rate of less than $25 \%$ (11). As gastrointestinal organs are under autonomic nervous control, associations between the nervous system and gastric cancer could be presumed. This study aimed to assess the role of the SNS and $\beta$-adrenergic pathway in the progression and metastasis of gastric cancer. Using immunohistochemical staining, we evaluated the density of SNS and expression of $\beta$-AR in surgically resected 
specimens of gastric cancer and then investigated whether they correlate with clinicopathological characteristics of gastric cancer. Furthermore, to confirm previous data showing neuronal influence on tumor progression through promoting angiogenesis and lymphangiogenesis (3), we investigated whether the blood and lymphatic vessel densities are associated with SNS density and/or $\beta$-AR expression status of gastric cancer.

\section{Materials and Methods}

Patients and tissue samples. This study (2017-01-003) was reviewed and approved by the Institutional Review Board of Kyung Hee University at Gangdong, Seoul, Republic of Korea. Patients who underwent radical gastrectomy for gastric cancer from January 2006 to December 2013 were included; those with small-sized $(<1 \mathrm{~cm})$ tumors or a history of other malignancy were excluded. Data on the clinicopathological characteristics of the patients, including age, sex, histological type, histological grade, tumor location, tumor size, pathological tumor stage (pT), pathological node stage $(\mathrm{pN})$, stage group, and recurrence were collected from the electronic medical records.

Immunohistochemistry. We collected $4-\mu \mathrm{m}$ tissue sections from gastric cancer specimens, and immunohistochemical staining was performed using the Bond Polymer Intense Detection system (Vision BioSystems, Victoria, Australia) according to the manufacturer's recommendations (12-15). In brief, 4- $\mu \mathrm{m}$ sections of formalin-fixed, paraffin-embedded tissue were deparaffinized with Bond Dewax Solution (Vision BioSystems), and an antigen-retrieval procedure was performed using the Bond ER Solution (Vision BioSystems) for $30 \mathrm{~min}$ at $100^{\circ} \mathrm{C}$. Endogenous peroxidases were quenched by incubating the tissues with hydrogen peroxide for $5 \mathrm{~min}$. The sections were incubated for $15 \mathrm{~min}$ at ambient temperature with primary antibodies against tyrosine hydroxylase $(\mathrm{TH} ; 1: 1,000$, polyclonal, Abcam, Cambridge, MA, USA), $\beta 1$-AR (1:200, polyclonal, Abcam), $\beta 2$-AR (1:100, polyclonal, Abcam), CD31 (1:40, clone JC70A, Dako, Glostrup, Denmark), and D2-40 (1:50, clone D2-40, Dako). A biotinfree polymeric horseradish peroxidase-linker antibody conjugate system was used with a Bond-maX automatic slide stainer (Vision BioSystems), and slides were visualized using $1 \mathrm{mM} \mathrm{3,3'-}$ diaminobenzidine, $50 \mathrm{mM}$ Tris-hydrogen chloride buffer ( $\mathrm{pH} 7.6$ ), and $0.006 \%$ hydrogen peroxide. The sections were counterstained with hematoxylin. The slides were dehydrated following a standard procedure and sealed with coverslips. To minimize the inter-assay variation, positive and negative control samples were included in each run. Immunohistochemical staining was independently analyzed by two board-certified pathologists who were blinded to the clinicopathological data. Disagreements between the two pathologists were resolved by consensus.

SNS density. TH is a rate-limiting enzyme required for biosynthesis of SNS neurotransmitters (16). TH immunoreactivity was used to measure the SNS density that was evaluated both within and around the tumor areas (intra- and peritumoral SNS, respectively; Figure 1). The areas of $\mathrm{TH}$-positive sympathetic nerves per square millimeter were determined by averaging the areas of $\mathrm{TH}$-stained nerve fibers in hot spots. An average of 5 digitally imaged, individual hot spots were obtained on each immunostained slide. A total of 590 hot-spot images were obtained under a $100 \times$ original
Table I. Baseline clinicopathological characteristics of patients with gastric cancer.

\begin{tabular}{|c|c|}
\hline Characteristics & Number of cases (\%) \\
\hline \multicolumn{2}{|l|}{ Age (years) } \\
\hline$\geq 61$ & $41(35.7)$ \\
\hline$<61$ & $74(64.3)$ \\
\hline \multicolumn{2}{|l|}{ Gender } \\
\hline Man & $81(70.4)$ \\
\hline Woman & $34(29.6)$ \\
\hline \multicolumn{2}{|l|}{ Tumor size $(\mathrm{cm})$} \\
\hline$\geq 5$ & $49(42.6)$ \\
\hline$<5$ & $66(57.4)$ \\
\hline \multicolumn{2}{|l|}{ Lauren classification } \\
\hline Intestinal & $96(83.5)$ \\
\hline Diffuse & $7(6.1)$ \\
\hline Mixed & $12(10.4)$ \\
\hline \multicolumn{2}{|l|}{ Histological grade } \\
\hline Well differentiated & $11(9.6)$ \\
\hline Moderately differentiated & $42(36.5)$ \\
\hline Poorly differentiated & $62(53.9)$ \\
\hline \multicolumn{2}{|l|}{ Pathological tumor stage (pT) } \\
\hline pT1 & $25(21.7)$ \\
\hline pT2 & $16(13.9)$ \\
\hline pT3 & $58(50.4)$ \\
\hline pT4 & $16(13.9)$ \\
\hline \multicolumn{2}{|l|}{ Pathological node stage $(\mathrm{pN})$} \\
\hline pNO & $26(22.6)$ \\
\hline pN1 & $34(29.6)$ \\
\hline $\mathrm{pN} 2$ & $21(18.3)$ \\
\hline $\mathrm{pN} 3$ & $34(29.6)$ \\
\hline \multicolumn{2}{|l|}{ Stage group } \\
\hline IA & $13(11.3)$ \\
\hline IB & $12(10.4)$ \\
\hline IIA & $18(15.7)$ \\
\hline IIB & $20(17.4)$ \\
\hline IIIA & $21(18.3)$ \\
\hline IIIB & $16(13.9)$ \\
\hline IIIC & $15(13.0)$ \\
\hline \multicolumn{2}{|l|}{ Tumor location } \\
\hline Antrum/angle & $62(53.9)$ \\
\hline Body & $44(9.9)$ \\
\hline Cardia & $5(4.3)$ \\
\hline Not applicable & $4(3.5)$ \\
\hline \multicolumn{2}{|l|}{ Recurrence } \\
\hline Present & $29(25.2)$ \\
\hline Absent & $86(74.8)$ \\
\hline
\end{tabular}

magnification (BX43 Microscope, Olympus, Tokyo, Japan) and individually analyzed using ImageJ, a Java-based image processing program developed at the National Institutes of Health (Bethesda, MD, USA). In each case, the sum of the intra- and peritumoral SNS density was considered as the total SNS density.

$\beta$-AR expression. ARs are members of the $\mathrm{G}$ protein-coupled receptor superfamily that bind endogenous catecholamine (i.e., epinephrine and norepinephrine) $(17,18) . \beta$-AR is classically localized to the plasma membrane, but can also be found in the cytoplasm and/or Golgi upon overexpression. The staining intensity 


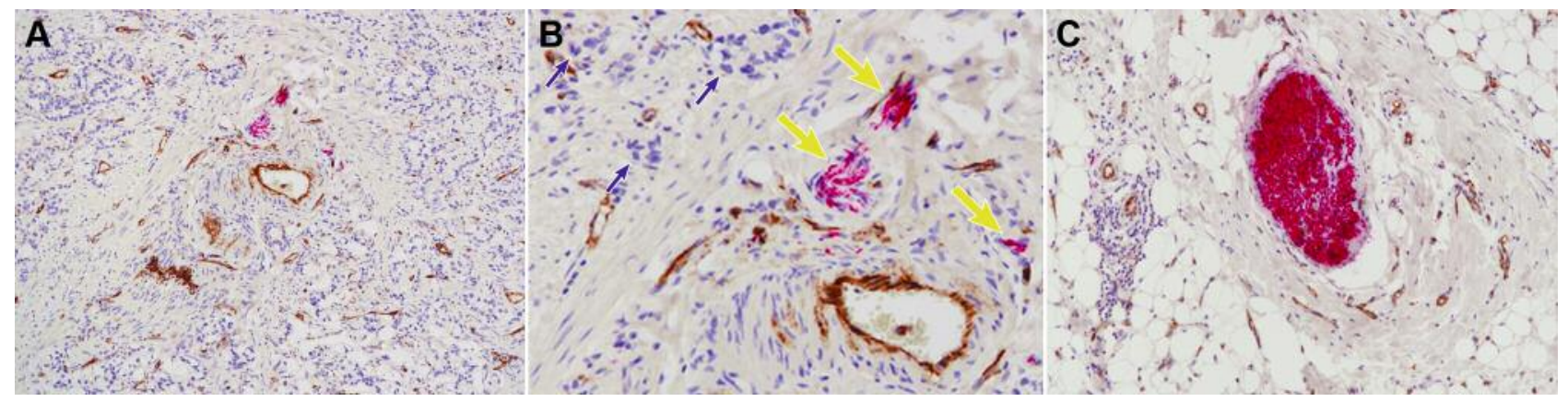

Figure 1. Representative photomicrographs showing immunoreactivities for tyrosine hydroxylase (TH). A: Low-power magnification of TH immunostaining in the intratumoral region. B: High-power magnification of image A. Red-stained nerve fibers (larger, yellow arrows) are those of the sympathetic nervous system. The gastric cancer cell nuclei (smaller, blue arrows) are counterstained with hematoxylin. CD31 highlights the vascular endothelial cells (brown color). C: Medium-power magnification of TH immunostaining in the peritumoral region. Polymer method. Original magnification: $A, 40 x ; B, 200 x ; C, 100 x$.
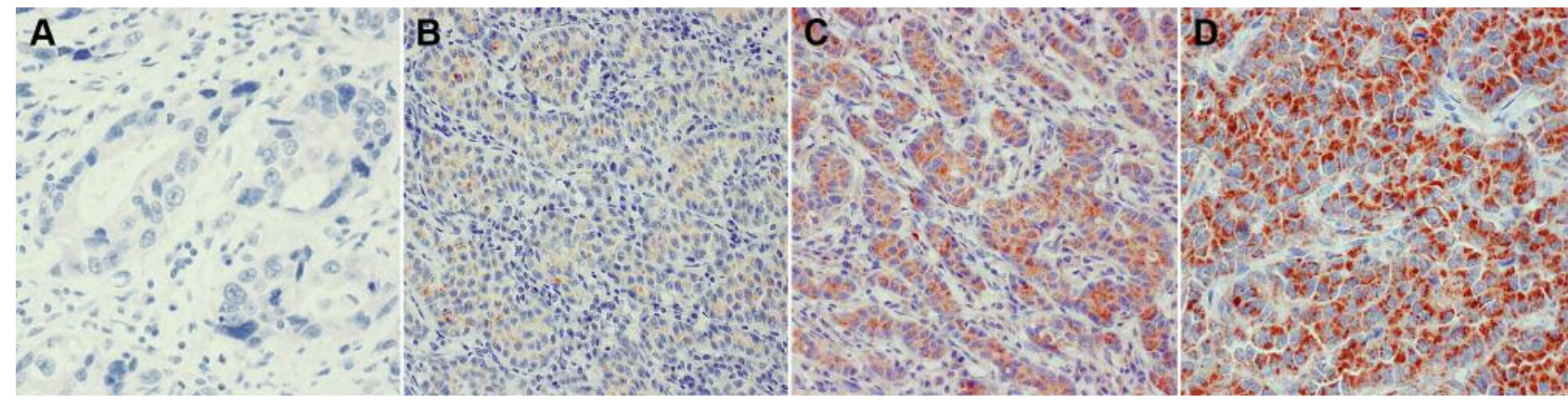

Figure 2. Representative photomicrographs showing staining intensities for $\beta 1$-adrenoceptor. $\beta 1$-AR is localized to the cytoplasm and/or Golgi zone. The staining intensity of $\beta 1-A R$ was assessed in gastric cancer cells on a scale of 0-3: A, 0 (negative); B, 1 (weak); C, 2 (moderate); D, 3 (strong). Polymer method. Original magnification: A, 400x; B, 100x; C, 100x; D, 200x.

of $\beta 1$ - and $\beta 2-\mathrm{AR}$ was assessed only in malignant tumor cells on a scale of $0-3$, with 0 indicating negative staining; 1 , weak; 2 , moderate; and 3, strong (Figure 2). The staining proportion which is defined as the percentage of positively stained tumor cells throughout the tumor, was also examined.

Blood and lymphatic and vessel densities. The representative tissue sections were stained with antibodies CD31 (vascular endothelial cell marker) and D2-40 (lymphatic endothelial cell marker) using aforementioned methods. Images of 5 individual hot spots were obtained from each immunostained slide. A total of 1,180 hot-spot images were obtained under a $100 \times$ original magnification and individually analyzed through manual counting by a single boardcertified pathologist who was blinded to the clinicopathological data.

Statistical analysis. One-way analysis of variance or Kruskal-Wallis test was used to examine the association between immunoreactivities and clinicopathological characteristics. Pearson's correlation analysis was performed to examine the relationship between the expression of proteins. Multivariate logistic regression analysis was used to identify the independent predictors. Statistical analyses were performed using IBM SPSS Statistics for Windows, version 20 (IBM Corp., Armonk, NY, USA). $p$-Value $<0.5$ was considered as statistically significant.

\section{Results}

Baseline characteristics. In total, 115 patients with gastric cancer were enrolled. Their baseline clinicopathological characteristics are summarized in Table I. The median patient age was 65 years (range $=32-93$ years), and $81(70.4 \%)$ and $34(29.6 \%)$ were men and women, respectively. In terms of Lauren classification, most $(96 / 115 ; 83.5 \%)$ cases were classified as intestinal-type gastric cancer. Seven $(6.1 \%)$ and $12(10.4 \%)$ cases were classified as diffuse- and mixed-type gastric cancer, respectively. In total, 11 (9.6\%), 42 (36.5\%), and $62(53.9 \%)$ cases were graded as well, moderately, and poorly differentiated gastric cancer, respectively. In terms of pT, 25 (21.7\%), 16 (13.9\%), 58 (50.4\%), and 16 (13.9\%) patients were diagnosed with pT1, pT2, pT3, and pT4, 

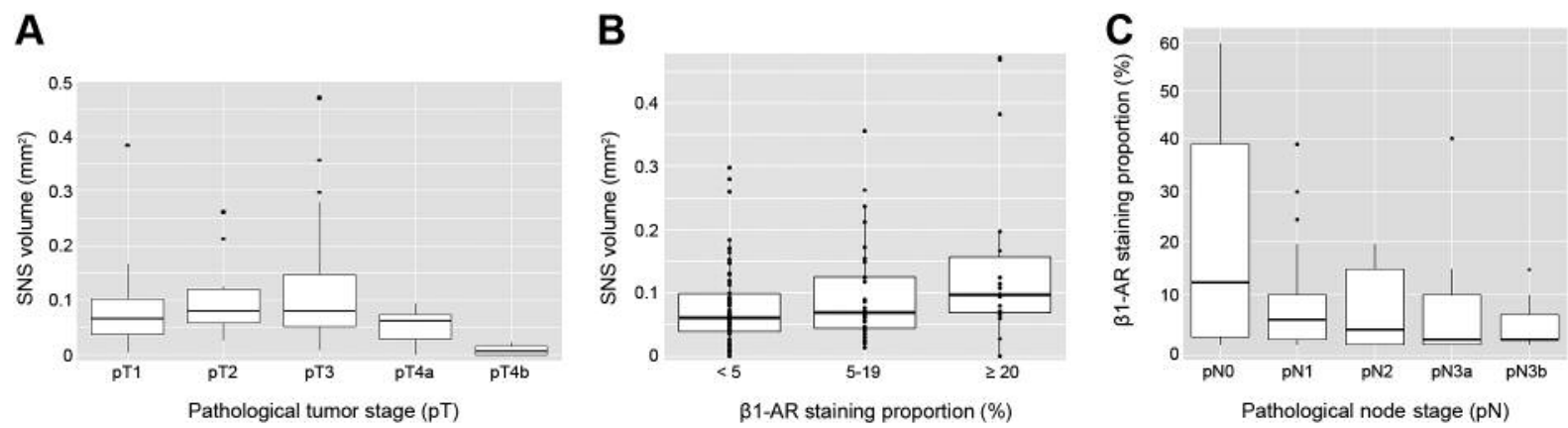

Figure 3. Relationship between sympathetic nervous system (SNS) density, protein expression, pathological tumor stage (pT), and pathological node stage $(p N)$. A: Relationship between SNS density and pT. SNS density of pT4a and pT4b tumors is significantly lower compared to that of pT1-3 tumors. B: Relationship between SNS density and $\beta 1$-adrenoceptor ( $\beta 1$-AR) staining proportion. The SNS density was positively correlated with the $\beta 1$-AR staining proportion. $C$ : Relationship between $\beta 1$-AR staining proportion and $p N$. $\beta 1$-AR staining proportion decreases as the number of metastatic lymph nodes increases.

respectively. Lymph node metastasis was identified in 89 (77.4\%) patients. In terms of $\mathrm{pN}, 26(22.6 \%)$ patients were classified to have pN0; 34 (29.6\%), pN1; 21 (18.3\%), pN2; $19(16.5 \%), \mathrm{pN} 3 \mathrm{a}$; and $15(13.0 \%), \mathrm{pN} 3 \mathrm{~b}$. The majority $(106 / 115 ; 92.2 \%)$ of tumor was located in the antrum/angle $(62 / 106 ; 58.5 \%)$ or body $(44 / 106 ; 41.5 \%)$ of the stomach. The median follow-up time was 36.4 months (range $=0.5$ 90.2 months). Twenty-nine (25.2\%) patients developed disease recurrence during the follow-up period. Nine (7.8\%) patients died of disease progression.

SNS density in gastric cancer. Our analysis of whether SNS density is associated with the clinicopathological characteristics of gastric cancer showed a significant difference in SNS density according to pT (Table II). Particularly, the SNS density of pT4a tumors $\left(0.0482 \pm 0.0228 \mathrm{~mm}^{2}\right)$ was significantly lower than that of pT1 $\left(0.0942 \pm 0.0572 \mathrm{~mm}^{2} ; p=0.045\right)$, pT2 $(0.1080 \pm 0.0752$ $\left.\mathrm{mm}^{2} ; p=0.024\right)$, and pT3 $\left(0.1097 \pm 0.0989 \mathrm{~mm}^{2} ; p=0.048\right)$ tumors. Furthermore, the SNS density of pT4b tumors $\left(0.0094 \pm 0.0112 \mathrm{~mm}^{2}\right)$ was significantly lower compared to that of pT4a tumors ( $p=0.029$; Figure 3A). Meanwhile, there were no significant differences in SNS density among pT1, pT2, and pT3 tumors (Table II). These findings indicate that pT4 gastric cancers exhibited significantly lower SNS density than pT1-3 gastric cancers, suggesting that lower SNS density is associated with increased invasiveness in gastric cancer. By contrast, other clinicopathological characteristics of gastric cancer did not significantly correlate with SNS density. Multivariate logistic regression analysis revealed that $\mathrm{pT}$ is the only independent predictive factor of SNS density.

$\beta 1-A R$ expression in gastric cancer. Cytoplasmic $\beta 1-\mathrm{AR}$ immunoreactivity was observed in the tumor cells. The perinuclear Golgi zone of the tumor cells was highlighted via $\beta 1-\mathrm{AR}$ immunostaining, and the results showed that $\beta 1-\mathrm{AR}$
Table II. Difference in sympathetic nervous system (SNS) density according to pathological tumor stage of gastric cancer.

\begin{tabular}{lllllll}
\hline $\begin{array}{l}\text { Pathological tumor } \\
\text { stage }(\mathrm{pT})\end{array}$ & $\begin{array}{c}\text { SNS density } \\
\left(\mathrm{mm}^{2}\right)\end{array}$ & & $p$-Value & \\
\hline pT1 & $0.0942 \pm 0.0572$ & & & & \\
pT2 & $0.1080 \pm 0.0752$ & 0.407 & & & \\
pT3 & $0.1097 \pm 0.0989$ & 0.558 & 0.947 & & \\
pT4a & $0.0482 \pm 0.0228$ & $0.045^{*}$ & $0.024^{*}$ & $0.048^{*}$ & \\
pT4b & $0.0094 \pm 0.0112$ & $0.036^{*}$ & $0.020^{*}$ & $0.046^{*}$ & $0.029^{*}$ \\
\hline
\end{tabular}

*Statistically significant.

expression was significantly correlated with SNS density in gastric cancer (Figure 3B). The staining proportion of $\beta 1$-AR expression was higher in tumors with higher SNS density. In addition, $\beta 1$-AR expression was significantly associated with $\mathrm{pN}$. Both the intensity and the proportion (Figure 3C) of $\beta 1$ AR immunostaining decreased significantly as $\mathrm{pN}$ increased from $\mathrm{pN} 0$ to $\mathrm{pN} 3$ ( $p=0.024$ and $p=0.003$, respectively; Table III). This indicates that $\beta 1$-AR expression has a significant inverse relationship with the number of metastatic lymph nodes in gastric cancer, suggesting that lower $\beta 1$-AR expression is associated with increased lymph node metastasis of gastric cancer. Meanwhile, other clinicopathological characteristics of gastric cancer failed to show a significant association with $\beta 1$-AR expression. Multivariate analysis revealed that $\mathrm{pN}$ independently predicted $\beta 1$-AR expression status in gastric cancer.

$\beta 2-A R$ expression in gastric cancer. $\beta 2-\mathrm{AR}$ expression was also observed in the cytoplasm of tumor cells. The peritumoral stromal cells did not react with $\beta 2$-AR protein. In contrast to $\beta 1$ AR protein expression, $\beta 2$-AR expression did not significantly 
Bae et al: Sympathetic Nervous System and $\beta$-Adrenoreceptor in Gastric Cancer

Table III. Difference in $\beta 1$-adrenoreceptor ( $\beta 1$-AR) expression according to pathological node stage (pN).

\begin{tabular}{|c|c|c|c|c|c|c|c|}
\hline \multirow[t]{2}{*}{$\mathrm{pN}$} & \multicolumn{4}{|c|}{$\beta 1-\mathrm{AR}$ staining intensity [number of cases $(\%)$ ] } & \multirow[b]{2}{*}{$p$-Value } & \multicolumn{2}{|c|}{$\beta 1$-AR staining proportion $(\%)$} \\
\hline & 0 & 1 & 2 & 3 & & Mean \pm SEM & $p$-Value \\
\hline $\mathrm{pNO}$ & $2(7.7 \%)$ & $1(3.8 \%)$ & $0(0 \%)$ & $23(88.5 \%)$ & $0.024 *$ & $21.0 \pm 21.2$ & $0.003^{*}$ \\
\hline $\mathrm{pN} 1$ & $7(20.6 \%)$ & $2(5.9 \%)$ & $3(8.8 \%)$ & $22(64.7 \%)$ & & $9.0 \pm 11.3$ & \\
\hline $\mathrm{pN} 2$ & $6(28.6 \%)$ & $0(0 \%)$ & $2(9.5 \%)$ & $13(61.9 \%)$ & & $7.4 \pm 7.7$ & \\
\hline $\mathrm{pN} 3 \mathrm{a}$ & $9(47.4 \%)$ & $1(5.3 \%)$ & $2(10.5 \%)$ & $7(36.8 \%)$ & & $6.1 \pm 8.9$ & \\
\hline $\mathrm{pN} 3 \mathrm{~b}$ & $2(13.3 \%)$ & $3(20.0 \%)$ & $0(0 \%)$ & $10(66.7 \%)$ & & $5.3 \pm 6.0$ & \\
\hline
\end{tabular}

*Statistically significant. SEM: Standard error of mean.

correlate with SNS density. Instead, there was a significantly inverse relationship between $\beta 2-\mathrm{AR}$ expression status and histological grade. In particular, poorly differentiated tumors showed significantly lower $\beta 2$-AR staining proportion than wellto moderately differentiated tumors ( $p=0.041$; Table IV). The majority $(24 / 33 ; 72.8 \%)$ of cases with $<5 \% \quad \beta 2$-AR staining proportion was poorly differentiated tumors. In line with this finding, $\beta 2$-AR staining proportion was significantly lower in tumors showing diffuse-type histology than those with intestinaltype histology ( $p=0.006$; Table IV). In multivariate analysis, however, histological grade and Lauren classification did not independently predict $\beta 2$-AR expression status in gastric cancer. Other clinicopathological characteristics of gastric cancer did not significantly correlate with $\beta 2$-AR expression status.

Blood and lymphatic vessel densities in gastric cancer. We performed immunohistochemical staining for CD31 and D240 to highlight blood and lymphatic vessels, respectively, but no significant relationship was found between blood or lymphatic vessel density and SNS density, $\beta$-AR expression status, or clinicopathological characteristics of gastric cancer.

\section{Discussion}

In this study, $\beta 1-\mathrm{AR}$ expression was significantly correlated with SNS density. Also, pT4 gastric cancers displayed a significantly lower SNS density than pT1-3 gastric cancers. Moreover, $\beta 1$-AR staining proportion decreased significantly as the number of metastatic lymph nodes increased. In addition, SNS density and $\beta 1$-AR expression were independent predictive factors of more advanced $\mathrm{pT}$ and $\mathrm{pN}$ in gastric cancer, respectively. These findings indicate that a reduction in SNS density and $\beta 1-\mathrm{AR}$ expression is associated with an aggressive behavior of gastric cancer including invasiveness and metastasis. However, our findings are in contrast to those of many previous studies demonstrating tumor-promoting roles of SNS and/or $\beta$-AR system. Liao et al. (19) demonstrated that $\beta$ adrenergic agonist enhanced proliferation of gastric cancer cell lines, whereas non-selective $\beta$-adrenergic antagonist decreased cellular proliferation in a concentration-dependent manner. In
Table IV. Difference in $\beta 2$-adrenoreceptor ( $\beta 2$-AR) staining proportion according to Lauren's classification and histological grade of gastric cancer.

\begin{tabular}{lcccc}
\hline Characteristic & \multicolumn{2}{c}{$\begin{array}{c}\beta 2-\text { AR staining proportion } \\
\text { [number of cases (\%)] }\end{array}$} & $p$-Value \\
\cline { 2 - 4 } & $<5 \%$ & $5-19 \%$ & $\geq 20 \%$ & \\
\cline { 2 - 4 } & & & & \\
Histological grade & $1(3.0)$ & $2(4.7)$ & $1(2.6)$ & $0.041^{*}$ \\
$\quad$ Well differentiated & $8(24.2)$ & $16(37.2)$ & $18(47.4)$ & \\
Moderately differentiated & $24(72.8)$ & $25(58.1)$ & $19(50.0)$ & \\
$\quad \begin{array}{l}\text { Poorly differentiated } \\
\text { Lauren classification }\end{array}$ & $27(81.8)$ & $36(83.7)$ & $33(86.8)$ & $0.006^{*}$ \\
$\quad$ Intestinal & $1(3.0)$ & $2(4.7)$ & $3(7.9)$ & \\
Diffuse & $5(15.2)$ & $5(11.6)$ & $2(5.3)$ & \\
Mixed & & & & \\
\hline
\end{tabular}

*Statistically significant.

vivo clonidine increased tumor growth, and a2-adrenergic antagonists completely reversed this effect (20). However, we also found some previous studies investigating paradoxical effects of SNS on tumor development and progression. Luthy et al. (20) documented the inconsistent nature of adrenoceptor function in breast cancer. Some authors found that $\beta$-agonists significantly inhibited cancer cell proliferation and tumor growth in mice, and others reported that $\beta$-blockers significantly reduced DNA synthesis. Pinero et al. (21) reported that $\beta$ adrenergic inhibition resulted in increased cellular proliferation and tumor growth in breast cancer. They also revealed that adrenalin seems to either increase or decrease proliferation of breast cancer cells, depending on its binding to $\alpha 2$ - or $\beta$-ARs (21). Considering the inverse relationship between $\beta 1$-AR expression and lymph node metastasis, it is also reasonable to assume that the parasympathetic nervous system (PNS) affects the progression and metastasis of gastric cancer. The SNS and PNS branches of the autonomic nervous system classically regulate bodily functions in opposite ways. It can be therefore assumed that the compensatory inhibition of SNS through PNS activation influences the progression and metastasis of gastric cancer. With regards to the contradictory results, opposite roles 
of identical $\beta$-adrenergic pathways in different types of cancer were noticed. In particular, our findings suggest that SNS and $\beta 1$-AR expression have tumor-inhibiting roles in gastric cancer.

A previous study by Magnon et al. (22) documented that $\beta 2-\mathrm{AR}$ in the peritumoral stromal cells played an important role in the initial phases of prostate cancer development by promoting tumor cell survival. In this study, we did not observe $\beta$-AR expression in the peritumoral stromal cells. Instead, consistent with the results for $\beta 1-A R$, there was a significantly inverse relationship between $\beta 2$-AR expression status and histological grade. Poorly differentiated tumors and those with diffuse-type histology exhibited significantly lower $\beta 2$-AR staining proportion. Our results suggest that $\beta 2$-AR expression is associated with cellular differentiation in gastric cancer and that the expression pattern of $\beta 2$-AR varies according to specific cancer cell types.

In conclusion, we performed a quantitative measurement of SNS density in human gastric cancer and examined the association of SNS density and $\beta$-AR expression status with the clinicopathological characteristics of gastric cancer. Lower SNS density and $\beta$-AR expression were found to be associated with aggressive oncogenic behavior including worse histological grade, advanced $\mathrm{pT}$, and increased lymph node metastasis. Our data suggest that the SNS and the $\beta$ adrenergic pathway are involved in negative regulation of gastric cancer progression. Further studies are required to identify molecular pathways for interpreting the possible contradictory effects of the autonomic nervous system.

\section{Acknowledgements}

This work was supported by a grant from Kyung Hee University in 2015 (KHU-20152150).

\section{References}

1 Cole SW, Nagaraja AS, Lutgendorf SK, Green PA and Sood AK: Sympathetic nervous system regulation of the tumour microenvironment. Nat Rev Cancer 15: 563-572, 2015.

2 Cole SW and Sood AK: Molecular pathways: beta-adrenergic signaling in cancer. Clin Cancer Res 18: 1201-1206, 2012.

3 Mancino M, Ametller E, Gascón P and Almendro V: The neuronal influence on tumor progression. Biochimica et Biophysica Acta (BBA)-Reviews on Cancer 1816: 105-118, 2011.

4 Magnon C: Role of the autonomic nervous system in tumorigenesis and metastasis. Mol Cell Oncol 2: e975643, 2015.

5 Tang J, Li Z, Lu L and Cho CH: beta-Adrenergic system, a backstage manipulator regulating tumour progression and drug target in cancer therapy. Semin Cancer Biol 23: 533-542, 2013.

6 Moretti S, Massi D, Farini V, Baroni G, Parri M, Innocenti S, Cecchi $\mathrm{R}$ and Chiarugi P: beta-adrenoceptors are upregulated in human melanoma and their activation releases pro-tumorigenic cytokines and metalloproteases in melanoma cell lines. Lab Invest 93: 279-290, 2013.

7 Palm D, Lang K, Niggemann B, Drell TLt, Masur K, Zaenker KS and Entschladen F: The norepinephrine-driven metastasis develop- ment of PC-3 human prostate cancer cells in BALB/c nude mice is inhibited by beta-blockers. Int J Cancer 118: 2744-2749, 2006.

8 Sloan EK, Priceman SJ, Cox BF, Yu S, Pimentel MA, Tangkanangnukul V, Arevalo JM, Morizono K, Karanikolas BD, Wu L, Sood AK and Cole SW: The sympathetic nervous system induces a metastatic switch in primary breast cancer. Cancer Res 70: 7042-7052, 2010.

9 Hasegawa H and Saiki I: Psychosocial stress augments tumor development through beta-adrenergic activation in mice. Jpn J Cancer Res 93: 729-735, 2002.

10 Inbar S, Neeman E, Avraham R, Benish M, Rosenne E and BenEliyahu S: Do stress responses promote leukemia progression? An animal study suggesting a role for epinephrine and prostaglandinE2 through reduced NK activity. PLoS One 6: e19246, 2011.

11 Jemal A, Center MM, DeSantis C and Ward EM: Global patterns of cancer incidence and mortality rates and trends. Cancer Epidemiol Biomarkers Prev 19: 1893-1907, 2010.

12 Jung YY, Sung JY, Kim JY and Kim HS: Down-regulation of BCell Translocation Gene 1 by Promoter Methylation in Colorectal Carcinoma. Anticancer Res 38: 691-697, 2018.

13 Joo JW, Kim HS, Do SI and Sung JY: Expression of Zinc Finger and BTB Domain-containing 7A in Colorectal Carcinoma. Anticancer Res 38: 2787-2792, 2018.

14 Do SI, Kim HS, Kim K, Lee H, Do IG, Kim DH, Chae SW and Sohn JH: Predictive value of sphingosine kinase 1 expression in papillary thyroid carcinoma. Anticancer Res 37: 5399-5405, 2017.

15 Do SI, Kim HS, Kim K, Lee H, Do IG, Kim DH, Chae SW and Sohn JH: Predictive and prognostic value of sphingosine kinase 1 expression in patients with invasive ductal carcinoma of the breast. Am J Transl Res 9: 5684-5695, 2017.

16 Tekin I, Roskoski R Jr., Carkaci-Salli N and Vrana KE: Complex molecular regulation of tyrosine hydroxylase. J Neural Transm (Vienna) 121: 1451-1481, 2014.

17 Xiao RP, Cheng H, Zhou YY, Kuschel M and Lakatta EG: Recent advances in cardiac beta(2)-adrenergic signal transduction. Circ Res 85: 1092-1100, 1999.

18 Kuznetsov V, Pak E, Robinson RB and Steinberg SF: Beta 2adrenergic receptor actions in neonatal and adult rat ventricular myocytes. Circ Res 76: 40-52, 1995.

19 Liao X, Che X, Zhao W, Zhang D, Bi T and Wang G: The betaadrenoceptor antagonist, propranolol, induces human gastric cancer cell apoptosis and cell cycle arrest via inhibiting nuclear factor kappaB signaling. Oncol Rep 24: 1669-1676, 2010.

20 Luthy IA, Bruzzone A, Pinero CP, Castillo LF, Chiesa IJ, Vazquez SM and Sarappa MG: Adrenoceptors: non conventional target for breast cancer? Curr Med Chem 16: 1850-1862, 2009.

21 Perez Pinero C, Bruzzone A, Sarappa MG, Castillo LF and Luthy IA: Involvement of alpha2- and beta2-adrenoceptors on breast cancer cell proliferation and tumour growth regulation. $\mathrm{Br}$ J Pharmacol 166: 721-736, 2012.

22 Magnon C, Hall SJ, Lin J, Xue X, Gerber L, Freedland SJ and Frenette PS: Autonomic nerve development contributes to prostate cancer progression. Science 341: 1236361, 2013.

Received November 29, 2018

Revised December 11, 2018 Accepted December 12, 2018 